\title{
Tratamento de fraturas patológicas tumorais diafisárias do úmero com haste intramedular rígida bloqueada estática - Experiência de 22 anos* $^{*}$
}

\section{Treatment of Pathological Humerus-Shaft Tumoral Fractures with Rigid Static Interlocking Intramedullary Nail - 22 Years of Experience}

\author{
Diogo Lino Moura $^{1}$ Filipe Alves $^{1}$ Rúben Fonseca ${ }^{1}$ João Freitas ${ }^{1}$ José Casanova ${ }^{1}$ \\ 1 Serviço de Ortopedia e Traumatologia, Centro Hospitalar e \\ Universitário de Coimbra, Coimbra, Portugal \\ Address for correspondence Diogo Lino Moura, Serviço de Ortopedia \\ e Traumatologia, Centro Hospitalar e Universitário de Coimbra, \\ Coimbra, Portugal (e-mail: dflmoura@gmail.com).
}

Rev Bras Ortop 2019;54:149-155.

\section{Resumo \\ Palavras-chave \\ - fraturas do húmero \\ - fixação intramedular de fraturas/ instrumentação \\ - fixação intramedular de fraturas/métodos \\ - fraturas espontâneas/ cirurgia \\ - metástase neoplásica}

Objetivo Estudo retrospectivo observacional em pacientes submetidos à fixação com haste intramedular de fratura patológica tumoral consumada ou iminente da diáfise do úmero em contexto de doença tumoral disseminada ao longo de 22 anos na mesma instituição.

Métodos Amostra com 82 pacientes e 86 fixações do úmero com haste intramedular rígida bloqueada estática não fresada anterógrada ou retrógrada.

Resultados Os tumores primários mais prevalentes foram carcinoma da mama $(30,49 \%)$, mieloma múltiplo $(24,39 \%)$, adenocarcinoma do pulmão $(8,54 \%)$ e carcinoma das células renais $(6,10 \%)$. O tempo médio de intervenção cirúrgica para fixação com haste foi $90,16 \pm 42,98$ minutos (40-135). Todos os pacientes referiram melhoria das queixas álgicas no nível do braço e velicou-se melhoria do score MSTS médio de $26 \%$ no pré-operatório para $72,6 \%$ na avaliação efetuada nos pacientes ainda vivos aos três meses de pós-operatório. A taxa de sobrevivência aos três meses após a cirurgia foi de $69,50 \%, 56,10 \%$ aos seis meses, $26,70 \%$ em um ano e $11,90 \%$ em dois anos. Nenhuma das mortes decorreu da cirurgia ou de complicações dela. Apenas se registaram quatro complicações relacionadas com a cirurgia, uma intraoperatória e três tardias, corresponderam a risco de complicações de $4,65 \%$.

Conclusão $\mathrm{O}$ uso de haste intramedular não fresada estática bloqueada (anterógrado ou retrógrado) no úmero é um método rápido, seguro, eficaz e com baixa morbilidade no tratamento das fraturas patológicas da diáfise umeral, garante fixação estável do braço e consequentemente melhora a funcionalidade e a qualidade de vida desses pacientes durante a sua curta expectativa de vida.

\footnotetext{
Trabalho desenvolvido no Serviço de Ortopedia e Traumatologia, Centro Hospitalar e Universitário de Coimbra, Coimbra, Portugal. Publicado originalmente por Elsevier Ltda.
}

received

August 10, 2017 accepted

October 31, 2017

published online

March 25, 2019
DOI https://doi.org/

10.1016/j.rbo.2017.10.012. ISSN 0102-3616.
Copyright $\odot 2019$ by Sociedade Brasileira License terms de Ortopedia e Traumatologia. Published by Thieme Revnter Publicações Ltda, Rio de Janeiro, Brazil 


\section{Abstract}

\section{Keywords}

- humeral fractures

- intramedullary fracture fixation/ instrumentation

- intramedullary fracture fixation/ methods

- spontaneous fractures/surgery

- neoplasm metastasis
Objective This was a retrospective observational study in patients submitted to intramedullary nail fixation after established or impeding pathological humerus-shaft tumoral fracture in the context of disseminated tumoral disease along 22 years of experience at the same institution.

Methods Sample with 82 patients and 86 humeral fixations with unreamed rigid interlocking static intramedullary nail by the antegrade or retrograde approaches.

Results The most prevalent primary tumors were breast carcinoma (30.49\%), multiple myeloma (24.39\%), lung adenocarcinoma (8.54\%), and renal cell carcinoma (6.10\%). The average surgical time was $90.16 \pm 42.98$ minutes (40-135 minutes). All of the patients reported improvement in arm pain and the mean Musculoskeletal Tumor Society (MSTS) score rose from $26 \%$ in the preoperative period to $72.6 \%$ in the evaluation performed in patients still alive 3 months after the surgery. The overall survival was $69.50 \% 3$ months after the surgery, $56.10 \%$ at 6 months, $26.70 \%$ at 1 year, and $11.90 \%$ at 2 years. No death was related to the surgery or its complications. There were only 4 surgery-related complications, 1 intraoperative and 3 late, corresponding to a $4.65 \%$ complication risk.

Conclusion Closed unreamed static interlocking intramedullary nailing (both in the antegrade or retrograde approaches) of the humerus is a fast, safe, effective, and low morbidity procedure to treat pathological fractures of the humerus shaft, assuring a stable arm fixation and consequently improving function and quality of life in these patients during their short life expectation.

\section{Introdução}

A patologia tumoral óssea, em particular a doença metastática óssea, tem vindo a registar um franco aumento da frequência nos últimos anos, os ossos longos são sede frequente desse tipo de lesão. $O$ úmero constitui o segundo local mais afetado por doença metastática no esqueleto apendicular (16\% a 20\% dos casos), segue-se ao fémur. ${ }^{1-3}$ As lesões metastáticas do úmero são mais frequentemente lesões líticas, condicionam fragilidade óssea e aumentam o risco de fratura, no entanto apenas $8 \%$ a $10 \%$ dessas evoluem para fratura consumada ou iminente. As fraturas patológicas do úmero constituem $16 \%$ a $39 \%$ de todas as fraturas patológicas dos ossos longos. ${ }^{1,4-7}$ Em regra geral, a fratura patológica da diáfise do úmero ocorre tardiamente na evolução da doença tumoral após um traumatismo menor ou espontaneamente durante atividades da vida diária e é um importante fator de prognóstico negativo e de morbilidade, causa dor, perda da função do membro e da independência do paciente, necessita muitas vezes de cuidados de apoio, de higiene e outros, o que reduz significativamente a qualidade de vida do paciente no seu curto tempo de vida expectável (de aproximadamente um ano após aparecimento de metástases ósseas em doença tumoral disseminada). ${ }^{1,2,4,5,8-12}$ A opção de tratamento conservador com imobilização nas fraturas patológicas do úmero demonstrou fracos resultados, com insuficiente redução da dor e pouca melhoria da função, sobretudo devido ao reduzido potencial de consolidação dessas fraturas devido aos efeitos biológicos e mecânicos do tumor. ${ }^{3-6,8,10,11}$ Face a isso, atualmente a cirurgia de osteossíntese é o tratamento gold-standard das fraturas patológicas diafisárias do úmero em contexto de doença tumoral disseminada, desde que o paciente não apresente contraindicações ao procedimento. ${ }^{1,3,10,11,13-17}$ Esse tratamento é essencialmente paliativo e tem como objetivos a estabilização eficaz imediata da fratura (não se pode contar nem esperar pela consolidação), o alívio da dor e a recuperação da mobilidade do membro, procura devolver independência funcional e qualidade de vida o mais precocemente possível, de preferência sem necessidade de mais intervenções cirúrgicas. A reduzida invasibilidade e a mínima agressão dos tecidos da técnica cirúrgica, bem como bons resultados tanto na estabilização imediata, alívio álgico e rápida recuperação funcional como na reduzida taxa de complicações decorrentes da cirurgia, tornaram os encavilhamentos intramedulares fechados do úmero a opção de eleição face a fraturas patológicas consumadas ou eminentes da sua diáfise. ${ }^{1,3-7,10,13-15}$ Apesar de não ser um tratamento inovador, os trabalhos efetuados acerca desse método específico de osteossíntese no contexto de fraturas patológicas do úmero e dos seus resultados são escassos e têm amostras pequenas. O objetivo deste estudo é analisar uma série de pacientes tratados com fixação intramedular com cavilha não fresada bloqueada estática em fraturas patológicas diafisárias do úmero e os resultados clínico-funcionais e radiográficos obtidos, tendo em particular atenção o tempo de sobrevivência pós-fixação e as complicações decorrentes da cirurgia.

\section{Material e métodos}

Desenvolvemos um estudo retrospetivo observacional em 101 pacientes consecutivos submetidos a estabilização paliativa do úmero com cavilha intramedular não fresada rígida 
aparafusada estática após diagnóstico de fratura patológica consumada ou iminente (essas últimas definidas como tendo score de Mirels ${ }^{18}$ superior a 8 ) da diáfise do úmero em contexto de doença tumoral disseminada ao longo de 22 anos na mesma instituição. Dezanove pacientes foram excluídos da amostra devido à obtenção insuficiente de dados sobre os mesmos, a amostra final é constituída por 82 pacientes. A avaliação incluiu colheita de dados demográficos, da localização e do tipo de neoplasia primária, da distribuição regional da lesão tumoral do úmero ao longo da sua diáfise, do tempo da intervenção cirúrgica, da via de encavilhamento efetuada, das dimensões da cavilha aplicada, do tempo de internamento, das complicações e do tempo de sobrevivência após cirurgia.

Foi efetuada uma avaliação clínica e funcional do membro superior com base na escala Musculo-Skeletal Tumor Society (MSTS). ${ }^{19}$ Fez-se ainda uma avaliação radiográfica do úmero, se considerou como definição de consolidação o desaparecimento da hipotransparência da fratura patológica e a presença de calo ósseo. Todos os encavilhamentos foram fechados, não fresados e o bloqueio da cavilha foi estático e efetuado com três ou quatro parafusos bicorticais proximais e distais. Todos os pacientes foram avaliados em consulta de ortopedia às seis semanas, três meses e seis meses ou até a data de falecimento. Também todos receberam ou continuaram tratamento antineoplásico de radioterapia, quimioterapia ou uma combinação de ambas. Alguns pacientes, em particular aqueles com tumores hipervasculares, tais como carcinoma das células renais, da tiroide e mieloma múltiplo fizeram angioembolização da lesão tumoral do úmero antes do encavilhamento. A data de falecimento dos pacientes, quando não disponível o registo de óbito, foi considerada como a data da primeira consulta ou do primeiro tratamento agendado aos quais o paciente faltou, sem remarcação ou novo comparecimento. Não foi possível recuperar todos os dados para todos os pacientes, o total dos casos sem essa informação foi sempre inferior a $20 \%$. Nessas situações os dados ausentes foram substituídos pelos valores médios dos restantes. Para o cálculo do tempo médio de intervenção cirúrgica correspondente ao encavilhamento, foram excluídos os oito pacientes (9,76\%) cuja cirurgia de encavilhamento foi efetuada no mesmo tempo operatório de outras intervenções cirúrgicas. Os dados foram tratados estatisticamente com recurso ao programa SPSSv23. Os valores são apresentados em número absoluto (n) e percentagens (\%), são preferidos como medida de tendência central a média e como medida de dispersão o desvio-padrão (valor mínimovalor máximo). As curvas de sobrevivência foram calculadas com o método de Kaplan-Meier. Todos os pacientes ou as famílias assinaram o termo de consentimento livre esclarecido e o presente estudo foi aprovado pela nossa instituição.

\section{Resultados}

Apresentamos uma amostra com 82 pacientes submetidos a 86 fixações do úmero com cavilha intramedular em contexto de fratura patológica consumada (-Fig. 1) ou iminente (-Fig. 2) por lesão tumoral. A idade média dos pacientes no momento de diagnóstico da fratura foi de $64,77 \pm 13,78$ (30-90). O diagnóstico no grupo dos homens foi feito com média de 61,96 anos, isto é, aproximadamente quatro anos mais cedo do que no grupo das mulheres, no qual a média foi de 66,23 anos. Aproximadamente $70 \%$ dos pacientes $(n=59)$ encontravam-se na sétima e oitava décadas de vida quando do diagnóstico de fratura patológica do úmero. A maioria dos pacientes era do sexo feminino $(69,51 \%, \mathrm{n}=57)$ e aproximadamente $90 \%$ das fraturas eram consumadas, restavam apenas oito fraturas iminentes que foram fixadas. A maioria das fraturas ocorreu à direita $(48,84 \%, \mathrm{n}=42)$ e quatro pacientes apresentavam fraturas bilaterais, três deles em simultâneo e um deles em tempos diferentes, o que equivale a 86 fraturas operadas em 82 pacientes. A distribuição

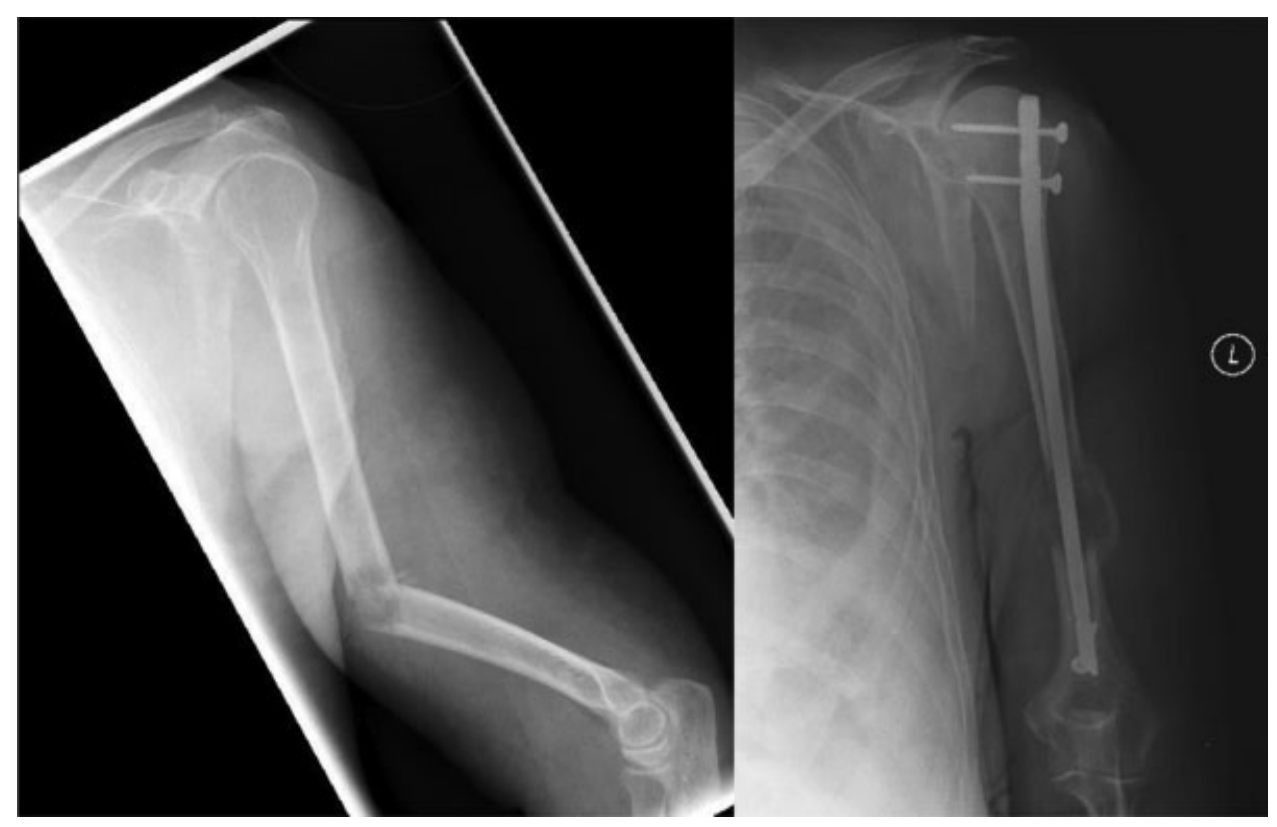

Fig. 1 Fratura patológica consumada da diáfise média do úmero e fixação com cavilha. 


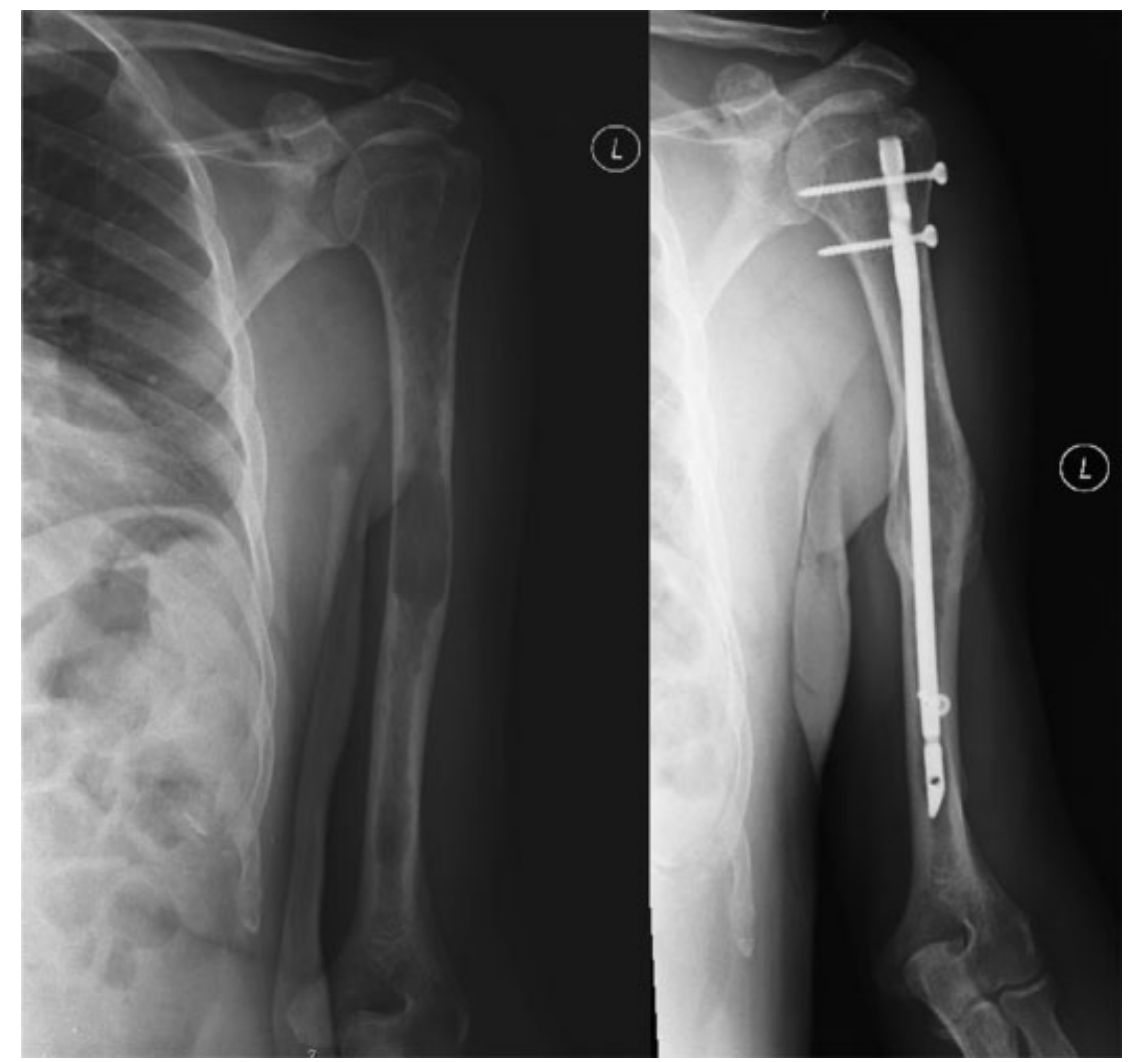

Fig. 2 Fratura patológica iminente da diáfise média do úmero e fixação com cavilha.

regional das lesões tumorais ao longo dos três terços da diáfise do úmero foi de forma decrescente ( - Fig. 3): diáfise média $(44,19 \%, \mathrm{n}=38)$, diáfise proximal $(36,05 \%, \mathrm{n}=31) \mathrm{e}$ diáfise distal $(19,77 \%, \mathrm{n}=17)$. Doze pacientes da amostra (14.63\%) tinham história prévia de fraturas patológicas em outros locais anatómicos, oito na coluna vertebral dorsolombar e quatro no fémur. Os tumores primários localizaram-se predominantemente nas seguintes áreas anatómicas por ordem decrescente (-Fig. 4): mama $(30,49 \%, n=25)$, sangue $(26,83 \%, \mathrm{n}=22)$, pulmão $(10,98 \%, \mathrm{n}=9)$, $\operatorname{rim}(8,54 \%$, $\mathrm{n}=7)$, próstata $(4,88 \%, \mathrm{n}=4)$, tiroide $(3,66 \%, \mathrm{n}=3)$, laringe $(2,44 \%, \mathrm{n}=2)$ e encéfalo $(2,44 \%, \mathrm{n}=2)$. O mieloma múltiplo é considerado nesta análise como tumor primário do sangue (hematopoiético). Em dois pacientes o tumor primário permaneceu oculto. As doenças tumorais que mais frequentemente provocaram as fraturas patológicas foram a doença metastática óssea e o mieloma múltiplo. Os tumores primários mais prevalentes foram o carcinoma da mama $(30,49 \%$,

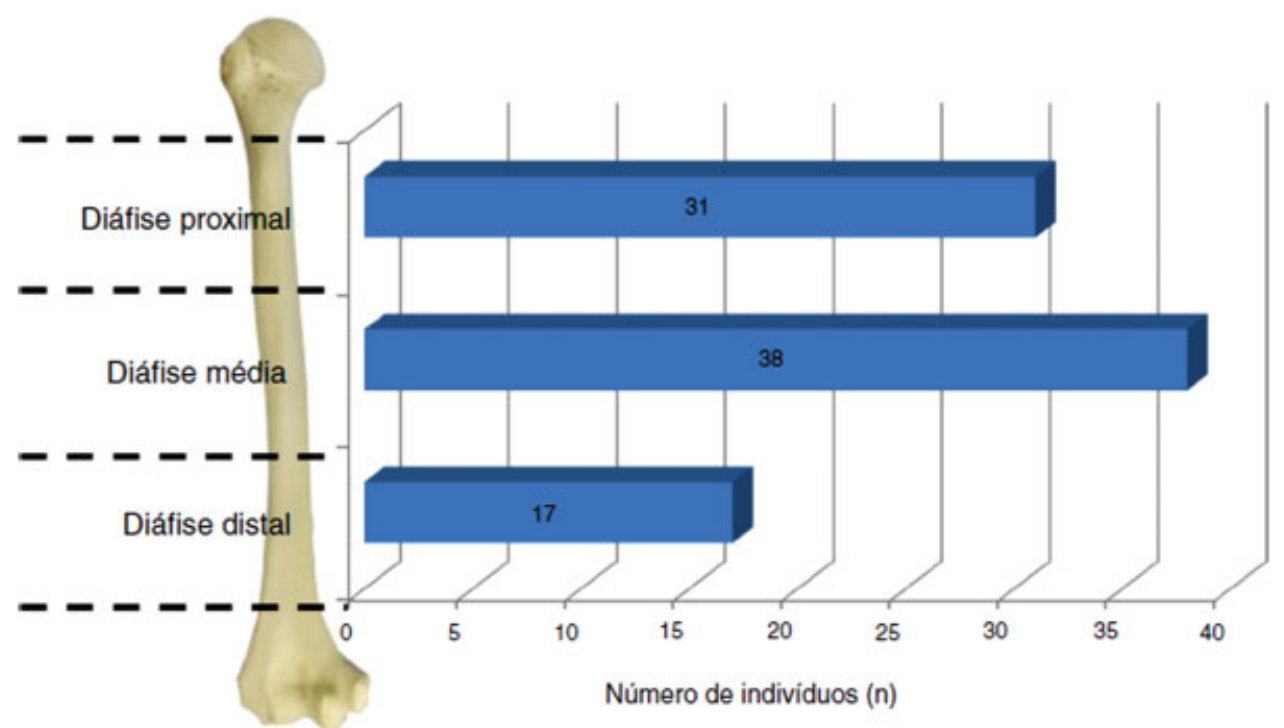

Fig. 3 Distribuição regional das lesões tumorais diafisárias do úmero. 


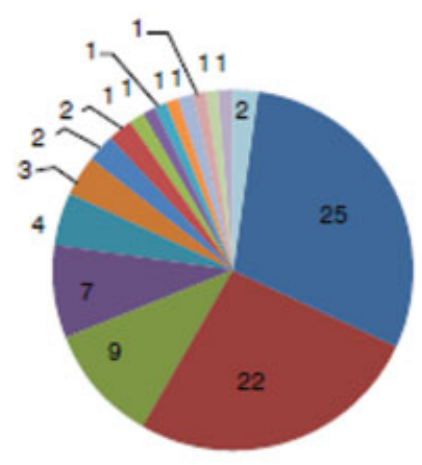

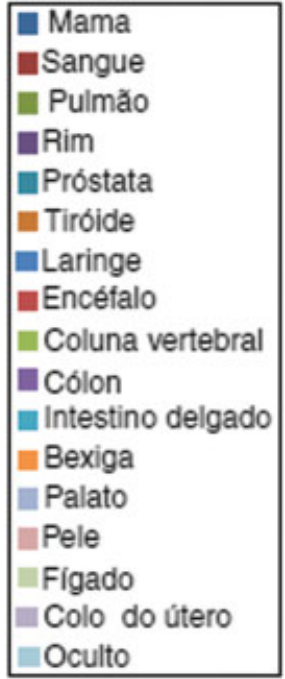

Fig. 4 Localização anatómica dos tumores primários. Nota: O mieloma múltiplo foi considerado como tumor primário do sangue (hematopoiético).

$\mathrm{n}=25)$, mieloma múltiplo $(24,39 \%, \mathrm{n}=20)$, adenocarcinoma do pulmão $(8,54 \%, \mathrm{n}=7)$ e o carcinoma das células renais $(6,10 \%, \mathrm{n}=5)$. 0 tumor primário mais frequente nas mulheres foi o carcinoma da mama (presente em $43,86 \%$ das mulheres) e nos homens o mieloma múltiplo (presente em $36 \%$ dos homens).

Na maioria dos pacientes $(86,59 \%)$ a fratura do úmero ocorreu com uma neoplasia base já diagnosticada previamente. No entanto, em 11 casos o diagnóstico oncológico foi apenas efetuado após a fratura patológica, assumiu-se essa como primeira manifestação da doença oncológica e motivou a pesquisa etiológica. $\mathrm{O}$ tempo médio entre o diagnóstico da neoplasia primária e o aparecimento da fratura patológica do úmero foi de $37,58 \pm 2,40$ meses. 0 tempo médio da inter- venção cirúrgica de fixação com cavilha foi de 90,16 \pm 42,98 minutos (40-135) e o tempo de internamento médio de $8,23 \pm 5,27$ dias (2-33). A maioria das fixações com cavilha ocorreu por via anterógrada $(68,60 \%, \mathrm{n}=59)$, as restantes foram efetuadas por via retrógrada $(31,40 \%, n=27)$. As dimensões das cavilhas usadas foram individualizadas caso a caso, levaram-se em conta as características anatómicas do úmero de cada paciente. Os diâmetros da cavilha mais frequentemente usados foram o $6,7 \mathrm{~mm}(66,28 \%, \mathrm{n}=57)$ e o $7,5 \mathrm{~mm}(16,28 \%, \mathrm{n}=14)$ e o comprimento mais aplicado foi o $250 \mathrm{~mm}(19,77 \%, \mathrm{n}=17$, intervalo 205-360). Foram mais frequentemente usados três parafusos de bloqueio (em $86,05 \%, n=74)$, nos restantes casos a cavilha foi bloqueada com quatro parafusos, dois proximais e dois distais.

Em termos clínico-funcionais, todos os pacientes referiram melhoria das queixas álgicas no nível do braço e verificou-se um aumento do score MSTS médio de 26\% para $72,6 \%$ na avaliação efetuada nos pacientes ainda vivos aos três meses de pós-operatório. Com a inclusão apenas dos 57 pacientes com fraturas consumadas que viveram um tempo igual ou superior a três meses após o encavilhamento, verificou-se uma taxa de consolidação radiográfica da fratura de $77,19 \%$ dos úmeros operados.

O tempo médio de sobrevivência após a cirurgia foi de $309,29 \pm 33,71$ dias e a mediana de 196 dias, com o maior número de mortes a ocorrer durante o 10 ano de pósoperatório. A taxa de sobrevivência aos 90 dias (três meses) após a cirurgia foi de 69,50\%, 56,10\% aos 180 dias (seis meses), 26,70\% aos 365 dias (um ano) e 11,90\% aos 730 dias (dois anos). Não ocorreram mortes no pós-operatório imediato, mas cinco pacientes $(6,10 \%)$ faleceram durante $o$ primeiro mês após a cirurgia. A análise de sobrevivência da amostra está ilustrada na - Fig. 5 através da curva de KaplanMeyer. Nenhuma das mortes decorreu da cirurgia ou de complicações associadas a ela.

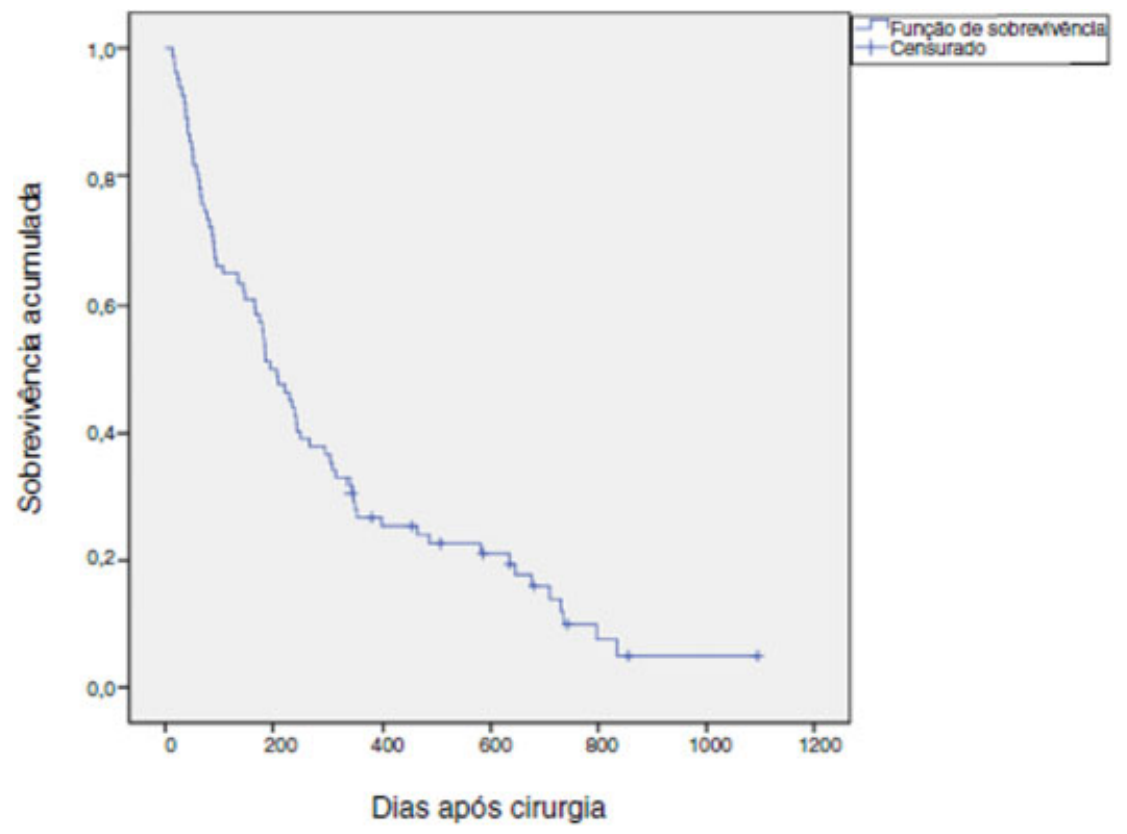

Fig. 5 Curva de Kaplan-Meyer a demonstrar a taxa de sobrevivência acumulada da amostra no tempo após encavilhamento do úmero em dias. 
Apenas se registaram quatro complicações relacionadas com a cirurgia, uma precoce intraoperatória e três tardias, corresponderam a um risco total de $4,65 \%$ de complicações para esse procedimento cirúrgico. As complicações foram: uma fratura iatrogénica do úmero distal numa via retrógrada, com necessidade de redução aberta e fixação com placa, parafusos e cerclagem; uma migração proximal da cavilha por perda da fixação dos parafusos proximais por crescimento da lesão tumoral, com necessidade de extração da cavilha e parafusos; um pullout de um dos dois parafusos de bloqueio proximal, com necessidade de extração isolada desse parafuso; e um síndrome de conflito subacromial por cavilha procidente, essas últimas três foram efetuadas por via anterógrada. Quando da extração da cavilha que migrou proximalmente constatou-se intraoperatoriamente que a fratura se encontrava consolidada. Não se verificaram quaisquer casos de lesão neurovascular, embolias, infeção, ossificação heterotópica, refratura ou outras. Não foi identificada qualquer diferença significativa no score funcional MSTS, no tempo de sobrevivência pós-cirurgia e na ocorrência de complicações de acordo com a idade, género, tipo de fratura consumada ou iminente e sua distribuição na diáfise umeral, tipo de tumor primário, via de encavilhamento, dimensões da cavilha e número de parafusos de bloqueio usados.

\section{Discussão}

Apesar de o tempo de vida expectável ter aumentado nas últimas décadas, a maioria dos pacientes que sofrem uma fratura patológica do úmero secundária a doença tumoral disseminada tem um tempo estimado de sobrevivência curto, de aproximadamente um ano, tal como demonstra o estudo atual, em que o tempo médio de sobrevivência após a cirurgia foi de 309,29 \pm 33,71 dias e a mediana de 196 dias. Como tal, nesses casos é imperativo que o tratamento escolhido tenha como prioridade o controlo imediato da dor e a restituição precoce da função, para minimizar a morbilidade associada e proporcionar uma melhor qualidade de vida em pacientes cujo estado geral se encontra comprometido. 1,2,4-6,8-12,20-22 Atualmente a abordagem gold-standard para fraturas patológicas da diáfise do úmero é a fixação com cavilha intramedular rígida e bloqueada proximal e distalmente de forma estática. ${ }^{1,3-7,10,13-15}$ Tanto quanto sabemos, este estudo é aquele com a amostra de maior dimensão em termos de tratamento de fraturas patológicas diafisárias do úmero com cavilha intramedular rígida na literatura em linguagem anglosaxónica. Tal como se verifica nesta série, cujos resultados são confirmados por estudos semelhantes, as cavilhas rígidas e bloqueadas estaticamente são um implante seguro e biomecanicamente eficaz (permitem fixação estável contra forças de angulação, rotação, distração e compressão) para a estabilização do úmero no contexto de fraturas patológicas diafisárias, permitem numa intervenção pouco demorada e num internamento hospitalar curto garantir a fixação do braço e consequentemente controlar a dor e restaurar a sua função, permitem mobilização imediata do membro e uma melhor qualidade de vida. Além disso, a cavilha permite fazer um bypass à lesão tumoral diafisária e assim obter uma fixação estável do implante em osso saudável proximal e distal, o que diminui o risco de falência de fixação. Garante também proteção profilática de um longo segmento do úmero contra novas fraturas e assim consequentemente contra nova cirurgia por eventual progressão da doença ou nova metástase. Ao tratar-se de uma técnica de redução fechada (sem abertura do foco de fratura), o encavilhamento permite também menor perda sanguínea, uma cicatrização mais rápida e menor risco de infeções, bem como a possibilidade de efetuar radioterapia adjuvante imediatamente após a cirurgia sem risco relevante de essa afetar a cicatrização, diminui assim o risco de progressão da doença tumoral e de consequentes sintomas e/ou falência da fixação, com necessidade de revisão cirúrgica. $^{1-10,13-17,23,24}$ Apesar de frequentemente esses pacientes estarem mais preocupados com o alívio álgico da fratura patológica do que com a função do membro, consideramos que a melhoria funcional obtida do score MSTS médio de $26 \%$ para $72,6 \%$ é bastante satisfatória. Apesar do previamente referido reduzido potencial de consolidação das fraturas patológicas, provavelmente a fixação estável proporcionada pela cavilha combinada com as terapêuticas antineoplásicas de radioterapia e quimioterapia permitiu que os índices de consolidação nesta amostra (avaliados apenas nos que sobreviveram mais de três meses após a cirurgia) fossem bastante satisfatórios (77,19\%), à semelhança do que verificaram Pretell et $\mathrm{al}^{1}$ (taxa de $80 \%$ ) e Atesok et $\mathrm{al}^{9}$ (taxa de $88 \%$ ) e que a incidência de complicações por falência da fixação se limitasse a um caso. ${ }^{1,2,9,11}$

Na nossa série apenas se verificaram quatro complicações e apenas uma delas intraoperatória de fratura iatrogénica do úmero quando da introdução da cavilha, a larga maioria da amostra obteve uma fixação estável do úmero durante todo o tempo de vida restante. Apesar de alguns estudos referirem níveis importantes de complicações com o encavilhamento de fraturas patológicas do úmero, em particular lesões nervosas quando da colocação dos parafusos de bloqueio, fraturas iatrogénicas quando da introdução da cavilha e falência da fixação dos parafusos de bloqueio, a maioria dos trabalhos está de acordo com o que verificámos na nossa amostra e refere também uma incidência de complicações cirúrgicas mínima. ${ }^{1,2,4-10,13,14,25}$ A via anterógrada tem sido mais associada a omalgias e rigidez do ombro por lesão iatrogénica da coifa dos rotadores e a via retrógrada mais associada a fraturas iatrogénicas do úmero quando da introdução da cavilha. 1,2,10,13 Apesar disso, neste estudo verificámos apenas uma de cada dessas complicações, o que atribuímos à técnica de encavilhamento usada e à experiência da equipa cirúrgica, consideramos que a maior parte das complicações operatórias relatadas na literatura pode diminuir claramente se a técnica de encavilhamento for a correta. A reduzida taxa de problemas no ombro devido ao ponto de entrada da cavilha anterógrada na nossa amostra poderá ser explicada pelas incisões únicas com lâmina fria do deltoide e coifa e seu encerramento adequado à proteção da coifa e deltoide quando da entrada da cavilha e ainda ao cuidado em não deixar a cavilha procidente. No entanto, devemos considerar que provavelmente também as menores expetativas funcionais e a maior tolerância álgica dos pacientes oncológicos terminais poderão ter 
algum impacto na reduzida prevalência de omalgias após encavilhamentos anterógrados do úmero. ${ }^{9}$ Consideramos ainda relevante a escolha adequada do diâmetro da cavilha tanto para prevenir fraturas iatrogénicas intraoperatórias como para aumentar a estabilidade da osteossíntese, é importante preencher a maior parte da cavidade medular do úmero de modo a diminuir a probabilidade de falências de fixação por instabilidade. Por sua vez, consideramos também que a ausência de embolias pulmonares na amostra justifica a manutenção do encavilhamento não fresado nos pacientes oncológicos com risco acrescido dessa complicação. ${ }^{2,26}$ As limitações deste estudo incluem a sua natureza retrospetiva, a ausência de dados de alguns pacientes e a perda de alguns pacientes no seguimento consequente à reduzida expetativa de vida na doença oncológica disseminada.

\section{Conclusão}

O encavilhamento intramedular não fresado estático bloqueado (anterógrado ou retrógrado) é um método de tratamento que preenche os requisitos adequados face a um paciente com curta expetativa de vida e com uma fratura patológica tumoral sintomática do úmero, na medida em que se trata de um método rápido, eficaz, seguro, com baixa morbilidade e que garante uma fixação estável e imediata do úmero, com consequente alívio das queixas álgicas, melhoria da funcionalidade e da qualidade de vida.

Conflitos de interesse

Os autores declaram não haver conflitos de interesse.

\section{Referências}

1 Pretell J, Rodriguez J, Blanco D, Zafra A, Resines C. Treatment of pathological humeral shaft fractures with intramedullary nailing. A retrospective study. Int Orthop 2010;34(04):559-563

2 Ofluoglu O, Erol B, Ozgen Z, Yildiz M. Minimally invasive treatment of pathological fractures of the humeral shaft. Int Orthop 2009;33(03):707-712

3 Dijkstra S, Stapert J, Boxma H, Wiggers T. Treatment of pathological fractures of the humeral shaft due to bone metastases: a comparison of intramedullary locking nail and plate osteosynthesis with adjunctive bone cement. Eur J Surg Oncol 1996;22(06):621-626

4 Sarahrudi K, Wolf H, Funovics P, Pajenda G, Hausmann JT, Vécsei V. Surgical treatment of pathological fractures of the shaft of the humerus. J Trauma 2009;66(03):789-794

5 Laitinen M, Nieminen J, Pakarinen TK. Treatment of pathological humerus shaft fractures with intramedullary nails with or without cement fixation. Arch Orthop Trauma Surg 2011;131(04):503-508

6 Gebhart M, Dequanter D, Vandeweyer E. Metastatic involvement of the humerus: a retrospective study of 51 cases. Acta Orthop Belg 2001;67(05):456-463

7 Redmond BJ, Biermann JS, Blasier RB. Interlocking intramedullary nailing of pathological fractures of the shaft of the humerus. J Bone Joint Surg Am 1996;78(06):891-896
8 Piccioli A, Maccauro G, Rossi B, Scaramuzzo L, Frenos F, Capanna R. Surgical treatment of pathologic fractures of humerus. Injury 2010;41(11):1112-1116

9 Atesok K, Liebergall M, Sucher E, Temper M, Mosheiff R, Peyser A. Treatment of pathological humeral shaft fractures with unreamed humeral nail. Ann Surg Oncol 2007;14(04):1493-1498

10 Frassica FJ, Frassica DA. Evaluation and treatment of metastases to the humerus. Clin Orthop Relat Res 2003(415, Suppl):S212-S218

11 Flemming JE, Beals RK. Pathologic fracture of the humerus. Clin Orthop Relat Res 1986;(203):258-260

12 Bauer HC, Wedin R. Survival after surgery for spinal and extremity metastases. Prognostication in 241 patients. Acta Orthop Scand 1995;66(02):143-146

13 Bauze AJ, Clayer MT. Treatment of pathological fractures of the humerus with a locked intramedullary nail. J Orthop Surg (Hong Kong) 2003;11(01):34-37

14 Spencer SJ, Holt G, Clarke JV, Mohammed A, Leach WJ, Roberts JL. Locked intramedullary nailing of symptomatic metastases in the humerus. J Bone Joint Surg Br 2010;92(01):142-145

15 Hunt KJ, Gollogly S, Randall RL. Surgical fixation of pathologic fractures: an evaluation of evolving treatment methods. Bull Hosp Jt Dis 2006;63(3-4):77-82

16 Bickels J, Dadia S, Lidar Z. Surgical management of metastatic bone disease. J Bone Joint Surg Am 2009;91(06):1503-1516

17 Capanna R, Campanacci DA. The treatment of metastases in the appendicular skeleton. J Bone Joint Surg Br 2001;83(04):471-481

18 Mirels $\mathrm{H}$. Metastatic disease in long bones. A proposed scoring system for diagnosing impending pathologic fractures. Clin Orthop Relat Res 1989;(249):256-264

19 Enneking WF, Dunham W, Gebhardt MC, Malawar M, Pritchard DJ. A system for the functional evaluation of reconstructive procedures after surgical treatment of tumors of the musculoskeletal system. Clin Orthop Relat Res 1993;(286):241-246

20 Hansen BH, Keller J, Laitinen M, Berg P, Skjeldal S, Trovik C, et al. The Scandinavian Sarcoma Group Skeletal Metastasis Register. Survival after surgery for bone metastases in the pelvis and extremities. Acta Orthop Scand Suppl 2004;75(311):11-15

21 Harrington KD. Orthopedic surgical management of skeletal complications of malignancy. Cancer 1997;80(8, Suppl): 1614-1627

22 Harrington KD, Sim FH, Enis JE, Johnston JO, Diok HM, Gristina AG. Methylmethacrylate as an adjunct in internal fixation of pathological fractures. Experience with three hundred and seventy-five cases. J Bone Joint Surg Am 1976;58(08):1047-1055

23 Townsend PW, Rosenthal HG, Smalley SR, Cozad SC, Hassanein RE. Impact of postoperative radiation therapy and other perioperative factors on outcome after orthopedic stabilization of impending or pathologic fractures due to metastatic disease. J Clin Oncol 1994;12(11):2345-2350

24 Damron TA, Rock MG, Choudhury SN, Grabowski JJ, An KN. Biomechanical analysis of prophylactic fixation for middle third humeral impending pathologic fractures. Clin Orthop Relat Res 1999;(363):240-248

25 Noger M, Berli MC, Fasel JH, Hoffmeyer PJ. The risk of injury to neurovascular structures from distal locking screws of the Unreamed Humeral Nail (UHN): a cadaveric study. Injury 2007; 38(08):954-957

26 Johnson JA, Berkshire A, Leighton RK, Gross M, Chess DG, Petrie D. Some basic biomechanical characteristics of medullary pressure generation during reaming of the femur. Injury 1995;26(07): 451-454 